PEDIATRIC UROLOGY

doi: $10.1590 / S 1677-55382010000600030$

\title{
Pelvic reduction during pyeloplasty for antenatal hydronephrosis: does it affect outcome in ultrasound and nuclear scan postoperatively?
}

Burgu B, Suer E, Aydogdu O, Soygur T

Division of Pediatric Urology, Department of Urology, Ankara University School of Medicine, Ankara, Turkey

Urology. 2010; 76: 169-74

Objective: To compare ultrasound (US) scan and nuclear renography findings in patients who underwent pyeloplasty with and without pelvic reduction in a randomized prospective study.

Methods: A total of 42 patients, all prenatally diagnosed with unilateral hydronephrosis, were included. Hydronephrosis was confirmed postnatally. Twenty patients were randomly selected to undergo pyeloplasty with pelvic reduction and 22 underwent pelvis-sparing pyeloplasty. Patients were evaluated with mercaptoacetyltriglycine-3 scans on the sixth month and US scans on the first, third, and sixth months, postoperatively. Mean follow-up was $37+/-5.6$ weeks. Statistical analyses were performed using chi-square test and significance was set as $\mathrm{P}$ $<.05$. Power analyses were performed by the NCSS-PASS program. Power value of 0.84 was calculated for a sample size of 42 .

Results: The anteroposterior pelvic diameter decreased significantly in the pelvic reduction group compared with pelvis-sparing group in the first- and third-month US scans. However, the difference was not significant in the sixth month. The improvements in the US findings for the pelvis-sparing group match with those of the pelvic reduction group later in the postoperative period. Pelvic reduction significantly improved the renal washout time $(\mathrm{T}(1 / 2))$ in mercaptoacetyltriglycine-3 renography when compared with pyeloplasty group without reduction at postoperative sixth month. Differential renal function was found to be unaffected from pelvic reduction.

Conclusions: Resolution of anteroposterior diameter in US scan is more prominent in the pelvic reduction group at earlier stages of the postoperative period. Although $\mathrm{T}(1 / 2)$ decreases more prominently in the pelvic reduction group, the utility of this procedure is still indecisive. This feature can reveal possible surgical failures earlier and strengthen the values of US and renography postoperatively.

\section{Editorial Comment}

This study is a prospective randomized trial looking at the effects of performing pelvic reduction during pyeloplasty. The authors used patients who had been antenatally diagnosed with unilateral hydronephrosis. Patients who demonstrated declining function in the hydronephrotic kidney with increasing pelvic dilation were recommended for pyeloplasty. They then randomized these patients to undergo either pelvic reduction at the time of pyeloplasty or not. They performed ultrasound at one, three, and six months postoperatively and repeated a MAG-3 diuretic renal scan at six months. They found a significant improvement in the degree of dilation in the pelvic reduction group at one and three months. However, at six months there was no significant difference between the two groups. Postoperative renal scans showed improved washout times in the pelvic reduction group although the authors point out that renal scans are unreliable indicators of obstruction. There was no difference in differential function at 6 months.

This study confirms the results of several other retrospective series suggesting that pelvic reduction is not necessary. What makes this study unique is that the patients were randomized in a prospective manner and followed according to a set protocol. In addition, this is a well-controlled population of infants at a mean of just over one year of age. For those who still favor pelvic reduction, the results suggest that persistent or worsening hydronephrosis on ultrasound following pelvic reduction might be a more worrisome sign of failure 
in the early post-operative period. It would certainly be interesting to see a follow-up study of these patients in another year or two to evaluate any changes in differential function of the kidneys over time.

\author{
Dr. M. Chad Wallis \\ Division of Pediatric Urology \\ University of Utah \\ Salt Lake City, Utah, USA \\ E-mail: chad.wallis@hsc.utah.edu
}

doi: $10.1590 / S 1677-55382010000600031$

\title{
Is staging beneficial for Fowler-Stephens orchiopexy? A systematic review
}

Elyas R, Guerra LA, Pike J, DeCarli C, Betolli M, Bass J, Chou S, Sweeney B, Rubin S, Barrowman N, Moher $\mathrm{D}$, Leonard $\mathrm{M}$

Queens University, Kingston, Ontario, Canada

J Urol. 2010; 183: 2012-8

Purpose: Fowler and Stephens showed that by dividing the spermatic vessels a high intra-abdominal testis could be placed in the scrotum. Testicular atrophy is a potential complication of this technique. We conducted a systematic review to determine whether single or 2-stage Fowler-Stephens orchiopexy results in better testicular viability.

Materials and Methods: We searched electronic databases, clinical trial registries and gray literature. We included reports describing boys younger than 18 years with a primary outcome of "testicular viability and position." We performed a meta-analysis using random effects models. Heterogeneity was assessed using forest plot and I(2) statistic.

Results: We identified 1,807 citations and included 61 articles. Single stage Fowler-Stephens orchiopexy was discussed in 9 articles, a 2-stage procedure in 36 and both approaches in 16. There were no randomized controlled trials, and most studies were cohort or case series. The pooled estimate of success rates was $80 \%$ for single stage Fowler-Stephens orchiopexy (95\% CI 75 to 86) and 85\% for 2-stage Fowler-Stephens orchiopexy (95\% CI 81 to 90$)$. The pooled odds ratio of single stage vs 2 -stage Fowler-Stephens orchiopexy was 2.0 (95\% CI 1.1 to 3.9) favoring the 2-stage procedure. There was no difference in the success rate between laparoscopic and open techniques in either single or 2-stage Fowler-Stephens orchiopexy. There was no evidence of asymmetry on the funnel plot. There were no complications reported with single stage, while ileus, hematoma and infection were the most common complications with 2-stage Fowler-Stephens orchiopexy.

Conclusions: Both techniques have a fairly high success rate but 2-stage Fowler-Stephens orchiopexy appears to carry a higher rate of success than the single stage approach ( $85 \%$ vs $80 \%$, OR 2 in favor of 2 -stage). Laparoscopic and open techniques had the same success rate. However, the level of evidence of the studies was low, and a study of a more robust design, such as a randomized controlled trial, should be performed.

\section{Editorial Comment}

This study is a meta-analysis of the English literature regarding Fowler-Stephens orchiopexy. The authors sought to determine if either a one-stage or a two-stage procedure had better outcomes. Out of over 1,800 citations they were able to include 61 articles. As one might imagine, their were no randomized controlled trials and the overall quality of these studies was average. Despite this, the statistical analysis demonstrated a slight advantage to a staged Fowler-Stephens orchiopexy with a success rate of $85 \%$ versus an $80 \%$ success rate in the single stage 
procedure. There was no significant difference noted between laparoscopic or open repair for either a single stage or a staged Fowler-Stephens orchiopexy. Those studies that looked at both a one-stage versus two-stage Fowler-Stephens orchiopexy were pooled together and favored a two-stage procedure with an odds ratio of 2 , although the numbers in many of these studies were quite small.

Although the strength of the data in this metaanalysis is not particularly strong it certainly favors a two-stage approach for these patients. Location of the testis and anatomy of the vas and vessels will vary from patient to patient. In addition, surgeon experience and comfort level with each of these procedures has to be taken into account. Having said that, however, this meta-analysis of the available literature gives the advantage to a staged procedure.

Dr. M. Chad Wallis

Division of Pediatric Urology

University of Utah

Salt Lake City, Utah, USA

E-mail: chad.wallis@hsc.utah.edu 